\title{
PYRAMIDAL TRACT RESPONSE TO CORTICAL STIMULATION IN THE RAT
}

\author{
Masahiro OHTA AND Nobuo TASHIRo \\ Department of Physiology, Faculty of Medicine, \\ Kyushu University, Fukuoka, Japan
}

ADRIAN and MORUZZI (1939) made the first record of the impulses in the pyramidal decussation as well as the potential waves in the cerebral cortex of the cat. They suggested that the high frequency discharges in the pyramidal tract were necessary to produce movement.

PATton and AMASSIAN (1952a, b, 1954) recorded the positive discharges (D and I waves) in the cortico-spinal tract evoked by cortical stimulation. They suggested that the first positive potential (D wave) was caused by direct excitation of the pyramidal tract neurons and the succeeding positive deflections (I waves) were evoked by the cortical synaptic pathways to the pyramidal tract neurons.

PHILlips (1956a, b) succeeded in making the first intracellular recordings from the cortical pyramidal tract cells of the cat and reported that the cortical stimuli which produced the movement evoked high frequency discharges in the Betz cells.

It is noteworthy that, in the rat, the antidromic cortical response to pyramidal tract stimulation was found to be distributed posteriorly wider than the so-called motor area (PorTer and SAnderson, 1964, OHTA, 1968). So it will be necessary to investigate the pyramidal tract response to cortical stimulation.

This paper is concerned with this purpose and the relation to the histological arrangement of the cortical pyramidal cells in the rat.

\section{METHODS}

Twenty adult rats, Wistar King $\mathrm{A}$, were used and anaesthetized with pentobarbital sodium (intraperitoneally, $30 \mathrm{mg} / \mathrm{Kg}$ initially and $5-10 \mathrm{mg} / \mathrm{Kg}$ in every succeeding hour).

The general procedure was almost the same as that described in the previous paper. The skull was removed with a dental bur and the exposed cortical hemisphere was covered with warm paraffin oil $\left(36-37^{\circ} \mathrm{C}\right)$ in a pool, surrounded by dental cement. The rectal temperature was maintained at 36 to 37 degree $\mathrm{C}$.

Received for publication September 1, 1967

太田雅博, 田代信維 
A bipolar stimulating electrode was made of silver wire, $0.5 \mathrm{~mm}$ in diameter, insulated in a glass pipette except for the tip. The interpolar distance was $1.0 \mathrm{~mm}$. The stimulating electrode was placed on the cortical surface which was stimulated electrically through an electronic stimulator-isolating unit (Nikkon MSE-2-Ja).

A bipolar recording electrode was made of silver wire, $0.1 \mathrm{~mm}$ in diameter and insulated in a glass pipette. The interpolar distance was $0.7 \mathrm{~mm}$. The stimulating electrode was inserted dorsally into the ipsilateral, medullary pyramidal tract, about $2.0 \mathrm{~mm}$ anterior from the pyramidal decussation.

After the experiment, the position of the tip of the recording electrode was confirmed histologically and the data taken when the electrode had not been in the correct position were discarded. Histological sections from the intact cerebral hemisphere were also made for the cortical pyramidal cells.

\section{RESULTS}

1. The form of the pyramidal tract response. The response recorded from the medullary pyramid ipsilateral to the stimulated cerebral hemisphere consisted of an initial positive wave and a succeeding one or a few positive potentials as shown in FIG. 1. The initial positive potential has a sharp rising phase and a sharp falling one. The time course was about $2 \mathrm{msec}$ and the mean value of the peak latencies was $2.08 \mathrm{msec}( \pm \mathrm{SD}=0.30 \mathrm{msec}, \mathrm{n}=278)$. This was thought to correspond with the D wave reported by PATTON and AMASSIAN (1952a, b, 1954, 1959) and seemed to correspond with the initial positive potential of the antidromic cortical response (PORTER and SANDERSON, 1964, OHTA, 1968).

The succeeding component was relatively small and often formed one positive wave but sometimes showed two or three waveforms. Sometimes there was no potential change following the $D$ wave. The mean value of the peak latencies of the first positive wave following the D wave was $4.93 \mathrm{msec}$ $( \pm \mathrm{SD}=0.92 \mathrm{msec}, \mathrm{n}=166$ ). This potential change seemed to correspond with the I wave of PATTON and AmAssian, (1952a, b, 1954, 1959).

These positive potential changes can be explained by the volume conductor theory (LORENTE DE Nó, R., 1953) and by the effects of nerve fiber injury made with the recording electrode. This problem will be discussed later.

2. Effect of altering the cortical stimulus site. The configuration of the pyramidal tract response changed with various cortical sites of stimulation. The maximum pyramidal tract response (both for the D and I waves) was obtained by stimulation at a cortical point, $8.0 \mathrm{~mm}$ anterior from the line connecting both ears and $3.0 \mathrm{~mm}$ lateral from the midline. The mean value of the $\mathrm{D}$ wave evoked by stimulation at that point was $141.9 \pm 20.5 \mu \mathrm{V}$ and the mean of the first I wave $\left(I_{1}\right)$ evoked by the same stimulation was $41.9 \pm 6.7 \mu \mathrm{V}$. As the cortical stimulus site was advanced in the frontal direction from the maximally effective point, the pyramidal tract response became smaller ; stimulating at the frontal pole, the $D$ wave showed about a half or one third of the 


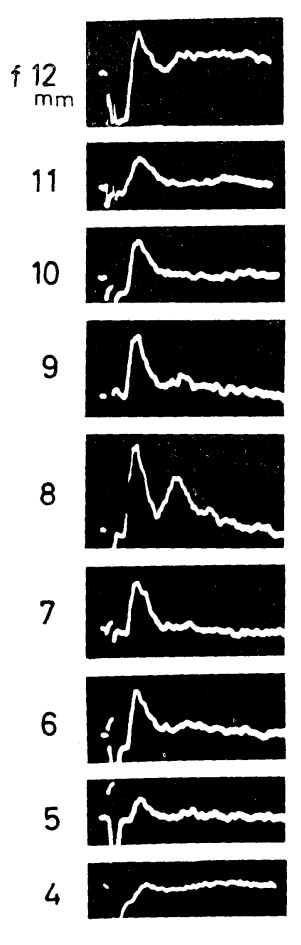

$5 \mathrm{msec}$
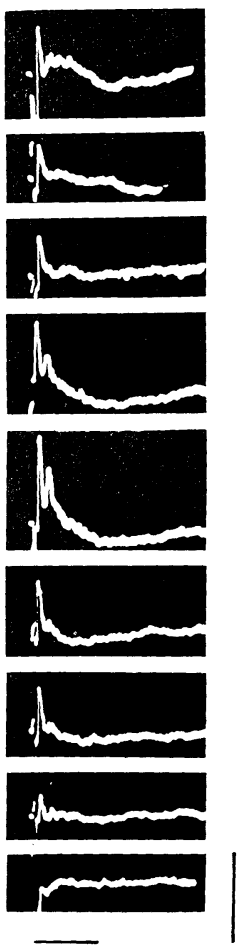

$20 \mathrm{msec} \quad 200 \mu \mathrm{V}$

FIG. 1. One case of the pyramidal tract response changes as the cortical stimulus site was altered in the fronto-occipital direction on a line $3.0 \mathrm{~mm}$ lateral from the midline. The left column has a fast sweep of recording, and the right column a slow sweep. The numbers at the left of each figure indicate the anterior distance from the line connecting both ears.

maximum response and the $I_{1}$ wave was reduced to about a third or a quarter of the maximum, as shown in the left diagram of FIG. 3. As the site of cortical stimulation advanced in the occipital direction from the maximum effective point, the response progressively decreased toward zero. The D wave decreased slightly and gradually until the point of stimulation reached $3.0 \mathrm{~mm}$ posterior from the maximum effective point and then abruptly decreased toward zero as the stimulus site was moved more occipitally. The $I_{1}$ wave decreased toward zero more rapidly than the $D$ wave as the site of stimulation advanced in the occipital direction from the maximum effective point.

The effect of varying the cortical stimulus site in the mediolateral direction on a line $8.0 \mathrm{~mm}$ anterior from the ear-line was also examined, and the pyramidal tract response was maximum both for the $D$ and $I_{1}$ waves when the 

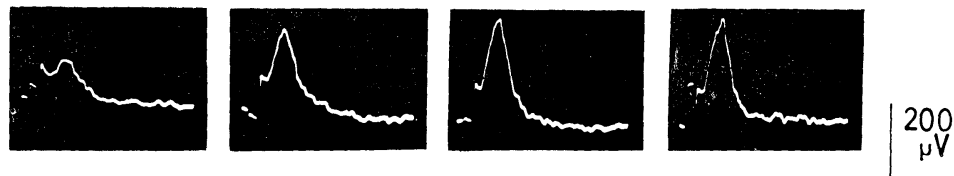

$5 \mathrm{msec}$
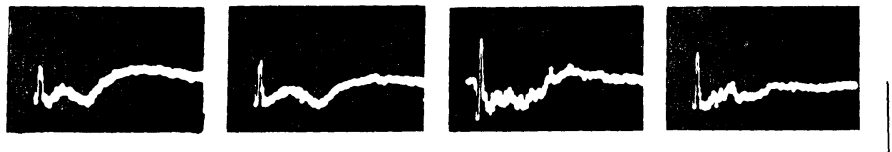

200

$20 \mathrm{msec}$

lat. $1 \mathrm{~mm}$ 2

3

4

FIG. 2. Two cases of the pyramidal tract response in which cortical stimuli were applied in the lateral direction on a line $8.0 \mathrm{~mm}$ anteric: from the ear-line. The numerals under each figure shows the lateral distance from the midline.

stimulus was applied at a point $3.0 \mathrm{~mm}$ lateral from the mid-line as shown in the right diagram of FIG. 3. The $\mathrm{D}$ wave, evoked by stimulation at a point $2.0 \mathrm{~mm}$ lateral from the mid-line, was slightly smaller than the maximum, the D wave evoked by stimulation at $4.0 \mathrm{~mm}$ lateral showed a little less amplitude, and the $\mathrm{D}$ wave evoked by stimulation at $1.0 \mathrm{~mm}$ lateral was the smallest and about a half of that at $3.0 \mathrm{~mm}$ lateral. $\mathrm{I}_{1}$ wave evoked by stimulation at $2.0 \mathrm{~mm}$ lateral was slightly smaller than that at $3.0 \mathrm{~mm}$ lateral and $\mathrm{I}_{1}$ evoked by stimulation at $1.0 \mathrm{~mm}$ lateral was even smaller. $\mathrm{I}_{1}$ evoked from $4.0 \mathrm{~mm}$ lateral was the smallest and was about half of that from $3.0 \mathrm{~mm}$ lateral.

FIG. 1 shows one case of the changes in the pyramidal tract response with variation of the stimulus site in the fronto-occipital direction on a line $3.0 \mathrm{~mm}$ lateral from the mid-line, with a fast sweep of recording in the left column and with a slow sweep of recording in the right column. The numbers indicate the distances of the stimulus site anterior from the line connecting both ears. FIG. 2 shows two cases of the pyramidal tract response changing with different stimulus sites in the medio-lateral direction on a line $8.0 \mathrm{~mm}$ anterior from the ear-line. The numbers below the figures indicate the lateral distance of stimulation from the midline. The upper column shows one case with a fast sweep of recording when the pyramidal tract response consisted of a $\mathrm{D}$ wave only. The lower column showed another case with a slow sweep of recording and in this case the I wave had a relatively long time-course with gradually rising and gradually falling phases. This slow I wave was composed of several smaller I waves overlapping one another.

FIG. 3 shows the mean amplitudes of the pyramidal tract response in eight cases changing with various cortical stimulus sites. The left diagram shows 

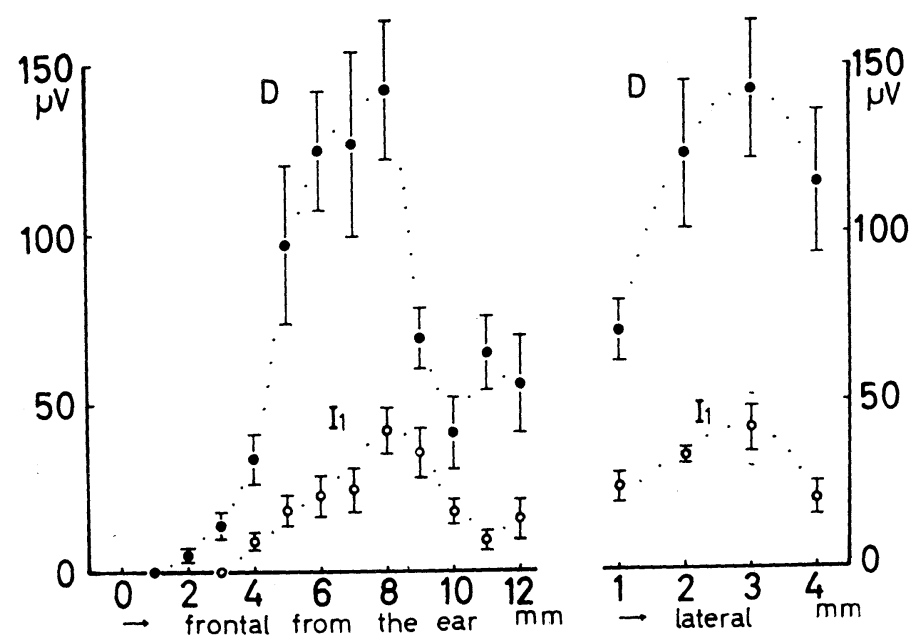

FIG. 3. Changes of the mean amplitude of the pyramidal tract response to stimulation applied at various cortical areas. The left diagram shows the effect of altering the cortical stimulus site in the fronto-occipital direction on a line $3.0 \mathrm{~mm}$ lateral from the midline and the right diagram indicates the effect of various stimulus sites in the lateral direction on a line $8.0 \mathrm{~mm}$ anterior from the ear-line. The black marks show the means of the D waves and the open circles for the means of the $I_{1}$ waves. The vertical bars indicate standard errors.

the effect of altering the stimulus site in the front-occipital direction on a line $3.0 \mathrm{~mm}$ lateral from the midline and the right diagram shows the effect of changing the stimulus site in the medio-lateral direction on a line $8.0 \mathrm{~mm}$ anterior from the ear-line. The black mark indicates the mean amplitude of the $D$ wave and the open circle shows the mean amplitude of the $I_{1}$ wave. The vertical bar shows the standard error.

This cortical representation of the pyramidal tract response corresponds fairly well with the cortical area responsible for motor effects in the rat, however, it is a little different from, and is localized more frontally than, the distribution of the antidromic cortical response to pyramidal tract stimulation. This is discussed later.

3. The relation of the response amplitude to the stimulus intensity. As the stimulus intensity was increased, the amplitude of the pyramidal tract response gradually increased up to the maximum, as shown in FIG. 4. The D wave had a lower threshold to cortical stimulation than the I wave.

As the stimulus intensity was gradually increased, the $D$ wave at first appeared as a very small potential but there was no I wave. Then with further increase of stimulus intensity, the $D$ wave nearly reached its maximum and the I wave appeared relatively small even at maximum stimulus intensity. As 

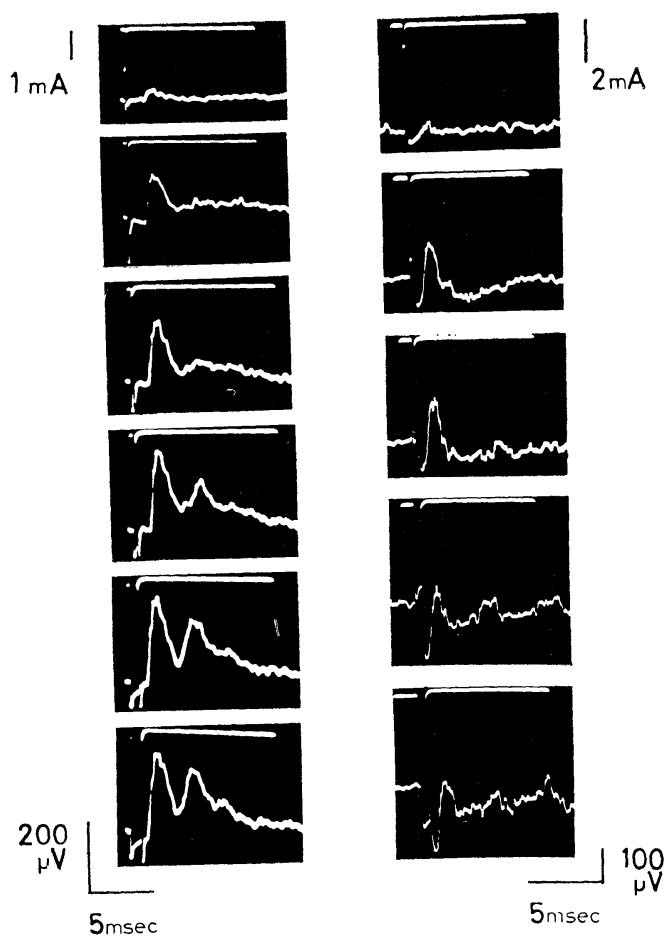

FIG. 4. Two cases of the pyramidal tract response in relation to the stimulus intensity. The cortical stimulation was applied at the maximally effective area of the cortical surface.

the stimulus was increased in duration, at maximum intensity, the I wave increased in amplitude and sometimes appeared repetitively in a few potentialwaves though the $D$ wave remained at its maximum amplitude.

FIG. 4 shows two cases of the pyramidal tract response to cortical stimulation at the maximally effective area, $8.0 \mathrm{~mm}$ anterior from the ear-line and $3.0 \mathrm{~mm}$ lateral from the midline. The upper vertical calibrations are of the stimulus intensity which was photographed in the upper beams of each record, the lower vertical bars are for the response amplitude and the lower horizontal bars are for the time scale.

FIG. 5 shows the response amplitude changing with the intensity and the duration of the cortical stimulus. Each response amplitude is shown as a percentage of the maximal value in each experimental series of three cases. The upper diagram indicates the amplitude change of the $D$ wave and the lower diagram the amplitude change of the $I_{1}$ wave. The $I_{1}$ wave had a two or three times higher threshold than the $\mathrm{D}$ wave. The horizontal scale shows the quantity of electricity (i.t, mA. msec). 

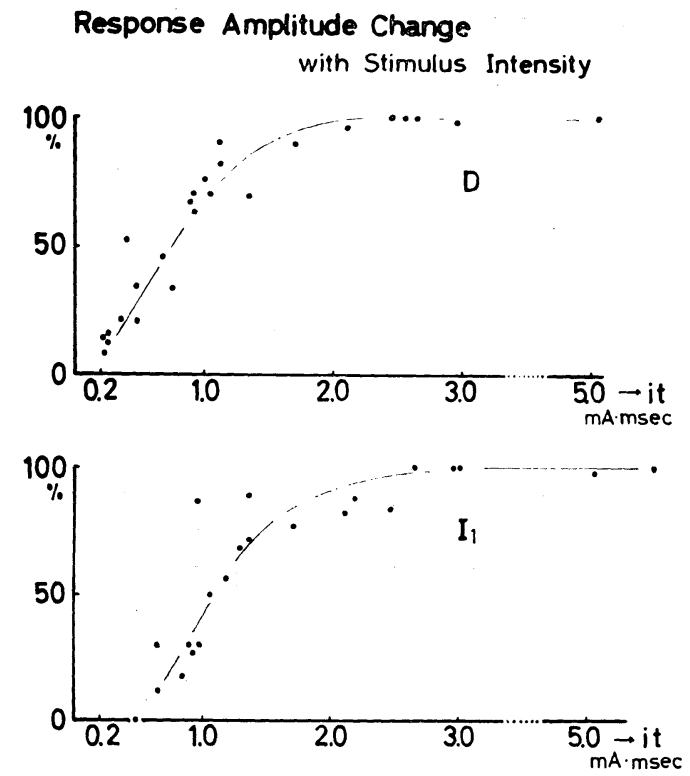

FIG. 5. Response amplitude change with the intensity and the duration of the cortical stimulus which was applied at the maximally effective site. The upper diagram shows the $\mathrm{D}$ wave and the lower one the $I_{1}$ wave. The numerals of the horizontal axis indicate the quantity of electricity. $(\mathrm{mA} \cdot \mathrm{msec}$ )

4. Effect of repetitive stimulation. As the frequency of cortical stimulation was increased up to $50 / \mathrm{sec}$, the I wave became larger in amplitude and appeared at gradually higher and higher frequencies, and the $\mathrm{D}$ wave became slightly larger in amplitude. However, when the frequency of stimulation increased up to $100 / \mathrm{sec}$, the I wave decreased both in amplitude and in frequency and often disappeared, but the $\mathrm{D}$ wave remained almost the same size as that evoked by $1 / \mathrm{sec}$ stimulation.

FIG. 6 shows one case of the change of the pyramidal tract response with increasing frequency of cortical stimulation, with a fast sweep of recording in the left column and with a slow sweep in the right column. In this case, stimulation at a frequency of $1 / \mathrm{sec}$ produced only a $\mathrm{D}$ wave and no I waves, but stimulation at a frequency of $5 / \mathrm{sec}$ evoked a $\mathrm{D}$ wave and a fairly late but relatively large I wave which had a peak latency of about $15 \mathrm{msec}$ after the cortical stimuli. Stimulation at a frequency of $10 / \mathrm{sec}$ produced a $\mathrm{D}$ wave and both a fast I wave and several late ones. As the frequency of stimulation was further increased up to $50 / \mathrm{sec}$, the fast I wave became larger in amplitude and had a slightly shorter peak latency, and the late I waves gradually increased in frequency but appeared with rather random peak latencies after 


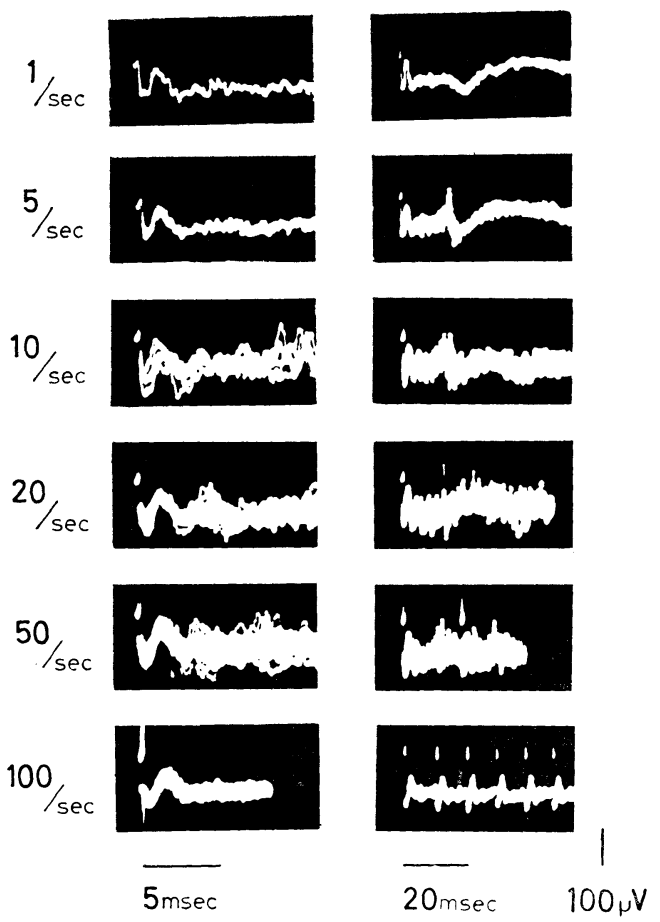

FIG. 6. One case of repetitive stimulation. The left column shows recordings with a fast sweep and the right column a slow sweep. Stimulation at a frequency of $5 / \mathrm{sec}$. evoked a D wave and a very late $I$ wave, and stimulation at a frequency of 10 to $50 / \mathrm{sec}$. produced a relatively large $D$ wave and fast $I\left(I_{1}, I_{2}\right.$, etc. $)$ waves, but stimulation at a frequency of $100 / \mathrm{sec}$. produced only a D wave.

the stimuli. However, stimulation at a frequency of $100 / \mathrm{sec}$ produced only a D wave with no I waves. Stimulation at this frequency might have excited both the excitatory presynaptic fibers and the inhibitory presynaptic fibers for the cortical pyramidal tract cells and therefore, the excitatory synaptic effects to these cells might have been suppressed by the inhibitory synaptic effects causing the absence of I wave. Stimulation at a frequency of under $50 / \mathrm{sec}$ might have mainly excited the excitatory presynaptic fibers for the cortical pyramidal tract cells.

In this case, stimulation at frequencies of between $20 / \mathrm{sec}$ and $100 / \mathrm{sec}$ produced movement of the forelimbs. With stimulation at frequencies of under $50 / \mathrm{sec}$, the D and repetitive I waves are thought to produce movements and with stimulation at a frequency of $100 / \mathrm{sec}$, repeatedly occurring $\mathrm{D}$ waves at $10 \mathrm{msec}$ interval seemed also to be effective. The impulses in the pyramidal tract occurring at a frequency higher than a certain level and with an amplitude 
larger than a certain level seem to be effective in producing movements. A single cortical stimulus with a stronger current in which large D and many I waves appeared was also effective in producing movements in some cases.

5. Effect of strychnine and procain applied to the cortical surface. Strychnine, GABA, d-tubocurarine and procain were applied to the cortical surface for one to ten min., and just after the period of application of each drug, the cortical surface was washed with warm Tyrode's solution and covered with warm paraffin oil and the pyramidal tract response to the cortical stimulation immediately recorded. The pyramidal tract response often gradually decreased in amplitude during these procedures, probably because of mechanical injury of the cortical surface.

GABA and d-tubocurarine had no effect on the pyramidal tract response to the cortical stimulation.

Strychnine, 1\% in Tyrode's solution, had scarcely any effect on the pyramidal tract response.

$2 \%$ strychnine had no effect on the D wave but produced and enhanced very late I waves, as shown in FIG. 7. This effect was most prominent with $2 \%$ strychnine applied for $1 \mathrm{~min}$. In five min. after washing the cortical surface, enhancement of these late I waves disappeared, but sometimes these enhanced

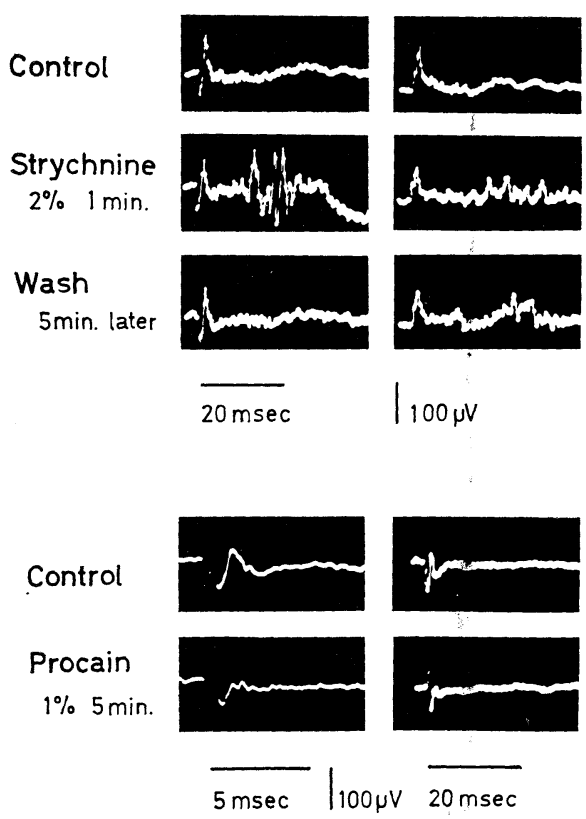

FIG. 7. Effects of strychnine and procain, applied on the cortical surface. Strychnine evoked late I waves. Procain blocked both $\mathrm{D}$ wave and I waves. 
late I waves persisted at a lower amplitude and decreased in number.

Strychnine is generally thought to be a depressor of the inhibitory system in the central nervous system which could fit the findings in this experiment. Strychnine might have blocked the inhibitory synaptic effects of cortical stimulation of the pyramidal tract neurons and the excitatory synaptic effects might have become apparent in enhancement of the late I waves.

Procain, $1 \%$ in Tyrode's solution applied for 2 min., slightly decreased both the $\mathrm{D}$ and I waves. The same procain solution for $5 \mathrm{~min}$. greatly decreased the $\mathrm{D}$ waves. This depression of the pyramidal tract response by procain persisted long after washing the cortical surface and the response could never recover its initial amplitude. Often respiration ceased and the animal died.

FIG. 7 shows the effect of strychnine in the upper diagrams and the effect of procain in the lower ones. In the left case of strychnine application, the late I waves are greatly enhanced in 15 to $20 \mathrm{msec}$ after the cortical stimulus, and in the right case, the late I waves are increased in 20 to $30 \mathrm{msec}$ after the cortical stimulus. The same calibrations for both the left and the right columns are shown under the diagrams. One case of procain is shown in the lower diagrams, the left with a faster sweep of recording and the right with a slower sweep. $1 \%$ procain greatly decreased the D wave and completely abolished the I wave which appeared in a small and relatively slow wave-form in this case. In this case, the depression of the pyramidal tract response persisted more than $15 \mathrm{~min}$. after washing the procain out from the cortical surface.

\section{DISCUSSION}

Comparing the pyramidal tract response with the antidromic cortical response, the $\mathrm{D}$ wave corresponds to the first positive component (PI) of the antidromic cortical response, and each peak latency is within SD from the other (OHTA, 1968). However, the I waves do not correspond to the second positive component (PII) of the antidromic cortical response. The first I wave had a peak latency of $4.93 \mathrm{msec}( \pm \mathrm{SD}=0.92 \mathrm{msec}, \mathrm{n}=166)$ and the peak latency of the PII was $3.58 \mathrm{msec}( \pm \mathrm{SD}=0.38 \mathrm{msec}, \mathrm{n}=150)$. This discrepancy is statistically significant. Moreover, the I waves showed larger facilitation with repetitive stimuli and therefore, it is reasonable to assume that the I waves may be evoked synaptically.

PATTON and AMASSIAN (1952a, b, 1954) found only the D wave but no I waves after decortication and they suggested the I waves were caused via the intracortical synaptic pathways. They recorded triphasic (positive-negativepositive) potentials from the ventral surface of the medullarly pyramidal tract and they found these potentials changed into monophasic positive waves when the recording electrode was inserted into the central axis of the pyramidal 
tract. These results were explained in terms of the volume conductor theory (LORENTE DE Nó, 1953) : in the tract nerve, approaching impulses were recorded as positive waves, impulses in the active site were recorded as negative potentials and far-going impulses were recorded as positive deflections; and monophasic positive potentials were recorded from the in jured fibers whose conduction was blocked by the recording electrode.

This effect of the recording electrode was also observed by LANCE (1954), who studied the cortico-spinal fibers between the medulla and the spinal cord, and he found two fiber groups with fast and slow conducting axons. However, it is not known whether or how much the slow conducting elements in the pyramidal tract take part in the pyramidal tract response to cortical stimulation.

PhILlips and his coworkers (1962a, b, c) observed the origin of the D and I waves directly with intracellular recordings. They observed how the cortical stimulus excited the Betz cells directly and synaptically and they succeeded in selectively exciting the Betz cells directly or synaptically by changing the polarity of the stimulating current. A surface positive current excited the Betz cells directly rather than synaptically, and a surface negative current tended to excite them synaptically. PHILlips and coworkers thought that the surface positive current might hyperpolarize the membrane of apical dendrites and would depolarize the cell bodies and the initial segment of the axons, however a surface negative current might depolarize the apical dendrites, but would not directly initiate an impulse in the Betz cells, and would excite the nerve fibers with synaptic contact to the Betz cslls.

These different effects of cortical stimulating currents of different polarity were comfirmed by LANDAu et al. (1964, 1965), Purpura and McMurtry (1965) and Gorman (1966).

The cortical representation of pyramidal tract response is a little different from, and slightly more frontally localized than distribution of the antidromic cortical response to pyramidal tract stimulation (PORTER and SANDERSON, 1964, OHтA, 1968). This discrepancy might be caused partly by the difference in techniques and partly by a difference between the excitability of the cortical pyramidal cells and that of the pyramidal tract fibers. The cortical representation of the pyramidal tract response seems to correspond fairly well with the density of the apical dendrites of the cortical pyramidal cells, regardless of size or depth from the cerebral surface.

In the frontal region, large pyramidal cells are lacking but many other pyramidal cells have well developed apical dentrites, which stretch obliquely and anteriorly toward the cortical surface. The apical dentrites are found most densely in the area from 5.0 to $8.0 \mathrm{~mm}$ anterior from the line connecting both ears. In the area less anterior than $5.0 \mathrm{~mm}$ from the ear-line, there are very few cortical pyramidal cells and they have very poorly developed apical dentrites. 
The large pyramidal cells are located most densely in the area from 5.0 to $7.0 \mathrm{~mm}$ anterior from the ear-line and their apical dentrites stretch slightly anteriorly toward the cortical surface. Therefore, the distribution of the cell bodies of the cortical pyramidal cells spreads slightly more posteriorly than the distribution of the apical dentrites. The electrical potential changes in the cell bodies of these cells may spread equally in all directions in the cerebral cortex but the electrical current applied on the cortical surface might spread along the apical dendrites of the cortical pyramidal cells. This arrangement of the apical dendrites of the cortical pyramidal neurons might cause the difference between the antidromic cortical response and the pyramidal tract response, and this might emphasize the functional significance of the apical dendrites of the cortical pyramidal tract cells.

The pyramidal tract response is thought to take a great part in the cortical motor effects, and in the rat, the cortico-spinal fibers might have multisynaptic effects rather than monosynaptic contacts to the spinal motoneurons since a single cortical stimulus failed to produce movement in many cases and repetitive stimuli were almost always effective. The major pathway from the cortico-spinal fibers to the spinal motoneurons seems to be multisynaptic in the cat (Scheibel and Scheibel, 1966, Lloyd, 1941), and in the monkey, the monosynaptic pyramidal connection exists and seems to make an important pathway to the spinal motoneurons (BERnHARd et al., 1953, Preston and Whitlock, 1960, 1961, LANDGREN et al., 1962a, b). The cortico-spinal effects project more directly upon the motoneurons as the phylogenetic scale is ascended from cats to primates (Scheibel and Scheibel, 1966). Therefore, it is reasonable to assume that the cortico-spinal effects may project more indirectly on the motoneurons in the rat than in the cat, and it may be under-standable that it is rather difficult to produce the movements in the rat by means of cortical stimulation. However, a study of the synaptic pathways from the corticospinal tract to the spinal motoneurons is necessary to understand the cortical motor functions in the rat.

\section{SUMMARY}

1. The pyramidal tract response to cortical stimulation was examined in twenty adult rats and was compared with the antidromic cortical response and with the histological sections of the cerebral cortex made by Bodian's method.

2. The pyramidal tract response consisted generally of two positive potentials, $\mathrm{D}$ and I waves. The $\mathrm{D}$ wave is caused by the direct excitation of the cortical pyramidal tract neurons and the I wave by the indirect or synaptic excitation of the same neurons. Sometimes there are a few I waves and sometimes there is no I wave.

3. The $\mathrm{D}$ wave has lower threshold for cortical stimulation and is less facili- 
tated by repetitive stimuli than the I waves.

4. The cortical representation of the pyramidal tract response corresponds fairly well with the cortical motor area but is slightly different from the distribution of the antidromic cortical response.

5. This is well explained by histological observation of the cortical pyramidal cells and the functional significance of the apical dendrites of these cells is emphasized.

\section{REFERENCES}

1) Adrian, E. D. And Moruzzi, G. Impulses in the pyramidal tract. J. Physiol., 97 : 153-199, 1939.

2) Bernhard, O.G., Bohm, E. And Petersen, I. Investigations on the organization of the corticospinal system in monkeys. Acta physiol. scand., 29: 79-105, 1953.

3) Gorman, A.L.F. Different patterns of activation of the pyramidal system elicited by surface anodal and cathodal cortical stimulation. J. Neurophysiol., 29 : 547-564, 1966.

4) Hern, J.E. C., Landgren, S., Phillips, C. G. and Porter, R. Selective excitation of corticofugal neurones by surface-anodal stimulation of the baboon's motor cortex. J. Physiol., 161: 73-90, 1962.

5) Lance, J. W. Pyramidal tract in spinal cord of cat. J. Neuro-physiol., 17: 253-270, 1954.

6) Landau, W.M., Bishop, G.H. And Clare, M.H. Site of excitation in stimulation of the motor cortex. J. Neurophysiol., 28 : 1206-1222, 1965.

7) Landgren, S., Phillips, C. G. And Porter, R. Minimal synaptic actions of pyramidal impulses on some alpha motoneurones of the baboon's hand and fore-arm. J. Physiol., 161: 91-111, 1962.

8) Landgren, S., Phillips, C. G. And Porter, R. Cortical fields of origin of the monosynaptic pyramidal pathways to some alpha motoneurones of the baboon's hand and fore-arm. J. Physiol., 161: 112-125, 1962.

9) Lloyd, D.P.C. The spinal mechanism of the pyramidal system in cats. J. Neurophysiol., 4 : 115-134, 1941.

10) Lorente De Nô, R. Conduction of impulses in the neurons of the oculomotornucleus. The Spinal Cord (London), 132-179, 1953.

11) Onta, M. Cortical motor function and the antidromic cortical response to stimulation of the medullary pyramidal tract in the rat. Jap. J. Physiol., $18: 100-124,1968$.

12) Patton, H.D. and Amassian, V.E. Responses in the corticospinal tract of cat and monkey. Fed. Proc., 11: 119, 1952.

13) Patton, H.D. and Amassian, V.E. Further observations on cortico-spinal discharges evoked by cortical stimulation. Amer. J. Physiol., 171: 756, 1952.

14) Patton, H.D. and Amassian, V.E. Single and multiple-unit analysis of cortical stage of pyramidal tract activation. J. Neurophysiol., 17:345-363, 1954.

15) Patton, H.D. and Amassian, V.E. The pyramidal tract: its excitation and functions. Handbook of Physiology, Washington, sec. I, vol. II, 837-861, 1959.

16) Phillips, C. G. Intracellular records from Betz cells in the cat. Quart. J. exp. Physiol., 41 : 58-69, 1956.

17) Phillips, C.G. Cortical motor threshold and the thresholds and distribution of excited Betz cells in the cat. Quart. J. exp. Physiol., 41: 70-84, 1956.

18) Phillips, C. G. And Porter, R. Unifocal and bifocal stimulation of the motor 
cortex. J. Physiol., 162 : 538, 1962.

19) Porter, R. And Sanderson, J.H. Antidromic cortical response to pyramidal tract stimulation in the rat. J. Physiol., 170: 355-370, 1964.

20) Preston, J.B. ANd Whitlock, D. G. Precentral facilitation and inhibition of spinal motoneurons. J. Neurophysiol., 23 : 154-170, 1960.

21) Preston, J.B. ANd Whitlock, D. G. Intracellular potentials recorded from motoneurons following precentral gyrus stimulation in primate. J. Neurophysiol., 24: 91-100, 1961.

22) Purpura, D.P. And McMurtry, J.G. Intracellular activities and evoked potential changes during polarization of motor cortex. J. Neurophysiol., 28 : 166-185, 1965.

23) Scheibel, M.E. ANd Scheibel, A.B. Terminal axonal patterns in cat spinal cord I: the lateral corticospinal tract. Brain Research, 2: 333-350, 1966. 